\title{
Contribution of camera-trapping to the knowledge of Abrocoma boliviensis
}

\author{
Carmen Julia Quiroga Pacheco ${ }^{1,2 *}$, Marisol Hidalgo-Cossio', and Ximena Velez-Liendo 3,4 \\ 1 Departamento de Mastozoología, Museo de Historia Natural Alcide d'Orbigny. Avenida Potosí 1458, Bolivia. Email: \\ carmen.j.pacheco@usn.no (CJQP), hidalgocossio.marisol@gmail (MHC). \\ ${ }^{2}$ University of South-Eastern Norway, Gullbringvegen 36, Bø i Telemark, Norway \\ ${ }^{3}$ The Recanati-Kaplan Centre, Wildlife Conservation Research Unit, Department of Zoology, University of Oxford. Tubney House, \\ Abingdon Road,9 X13 5QL. Tubney, United Kingdom. Email: x.velezliendo@gmail.com (XVL). \\ ${ }^{4}$ Conservation Department, North of England Zoological Society, Zoological Gardens Chester. Upton-by-Chester CH2 1EU. \\ Chester, United Kingdom. \\ *Corresponding author
}

Rare and endemic rodent species, such as the critically endangered Bolivian chinchilla rat, Abrocoma boliviensis, are infrequently captured using traditional trapping methods. Therefore, to determine their presence and abundance, indirect records (bird pellets, feces, bone remains) are often used. Although not a popular method for rodents, the incorporation of photography is known to provide precise data. Therefore, the objective of this report was to demonstrate the importance of using photographic records and occupancy models in the study of the distribution and ecology of a small-body species with high conservation value. We used photographic records obtained from 180 camera-trap nights in two areas of the Andean dry forests of Bolivia, during both wet and dry seasons. Data analysis include the estimates of presence probability of $A$. boliviensis in the study sites, using an exploratory occupancy analysis. The estimated presence probability of Abrocoma boliviensis for the study sites were $30.2 \%$ during dry season and $33.9 \%$ during wet season. However, due to the limited amount of photographic records for the species, it was not possible to accurately determine the factors affecting the species presence probability. Camera-trapping records and associated metadata provided new ecological and distribution data for the Bolivian chinchilla rat. In combination with information about threats that the species faces, our data may be useful in generating and applying conservation actions.

Roedores raros y endémicos como la críticamente amenazada rata chinchilla boliviana, Abrocoma boliviensis, son escasamente capturadas con el uso de trampas tradicionales. Contar con diferentes registros indirectos como egagrópilas, heces, restos de huesos y letrinas, aproximan a determinar la presencia de especies muy importantes. Los registros fotográficos, a pesar de no ser un método popularmente utilizado en roedores, se pueden considerar como datos precisos para el desarrollo de investigaciones. Por ello, el objetivo de este trabajo fue mostrar la utilidad de los registros fotográficos y modelos de ocupación en estudios sobre la distribución y ecología de especies de pequeño tamaño, pero con gran valor de conservación. Se utilizaron fotografías obtenidas a través de campañas de foto-trampeo durante 180 noches, en épocas seca y húmeda, en dos áreas de bosques secos andinos de Bolivia. Se analizó la probabilidad de presencia de A. boliviensis en el área de estudio a través de un análisis exploratorio mediante el uso de modelos de ocupación. Se determinó una probabilidad de presencia de la especie en el área de estudio del 30.2 \% para la época seca y de 33.9 \% para la húmeda. Debido a una limitada cantidad de registros fotográficos de A. boliviensis, no se pudo determinar con exactitud qué factores afectan la probabilidad de presencia de la especie. Los registros fotográficos obtenidos de foto-trampeo demostraron tener un potencial en las búsquedas de nuevas localidades donde reside la rata chinchilla boliviana. Esta nueva información obtenida acerca de especies únicas y elusivas como A. boliviensis aportan al conocimiento de su ecología, y por ende, derivan en la generación y aplicación de medidas para su protección y el de sus hábitats potenciales.

Keywords: Andean dry forests; Bolivian Chinchilla Rat; occupancy modeling; photographic records; rodents.

(c) 2020 Asociación Mexicana de Mastozoología, www.mastozoologiamexicana.org

\section{Introducción}

Dentro de los mamíferos, los roedores constituyen uno de los grupos más diversos y menos conocido (Burgin et al. 2018); debido a su actividad nocturna, conducta elusiva y baja probabilidad de captura el conocimiento sobre su comportamiento es muy escaso (Tarifa et al. 2009; Ferreguetti et al. 2018a; Thomas et al. 2020) en particular de aquellas especies amenazadas, endémicas o raras (Úbeda et al. 1994; Pacheco 2002; Tarifa y Aguirre 2009; IISE 2011; Law et al. 2016). La rata chinchilla boliviana, Abrocoma boliviensis (Rodentia: familia Abrocomidae), es una especie endémica para Bolivia y está categorizada como "En peligro crítico" tanto a nivel nacional (Tarifa y Aguirre 2009) como internacional (Bernal 2016). Además,
A. boliviensis es miembro de un grupo selecto de las 100 especies de mamíferos considerados con el más alto valor para la conservación por ser una especie evolutivamente distinta y globalmente amenazada (Isaac et al. 2007; EDGE 2019).

Bolivia presenta dos especies de Abrocomidae (Patton et al. 2015), A. cinerea, con amplia presencia en la región altiplánica de los Andes y A. boliviensis, considerada endémica de los Andes Centrales de Bolivia (Aguirre et al. 2009; Bernal 2016). A. boliviensis sólo se conoce a través de dos especímenes colectados en la localidad tipo, Comarapa, en 1926 y 1955 (Glanz y Anderson 1990), y posteriores esfuerzos para registrar a la especie en esta localidad resultaron fallidas (Glanz y Anderson 1990). En 
otras localidades de Bolivia se encontraron indicios de la presencia de la especie, a través de restos obtenidos en estómagos de serpientes y fotografías (Rivero et al. 2004; Tarifa etal.2009; Quinteros-Muñoz 2015; Hidalgo-Cossio etal. 2016). En los registros fotográficos, la especie es fácilmente identificable debido a sus características morfológicas, como la cola larga y peluda (Patton et al. 2015; Taraborreli et al. 2015); sumado a su tamaño, hocico corto y ancho, orejas grandes y redondas con una ligera muesca, patas gruesas y notorias respecto a otros roedores cricétidos (Glanz y Anderson 1990; Emmons 1999; Braun y Mares 2002). Por tanto, el uso de trampas cámara promete ser ideal para trabajar con esta especie, que, debido a su timidez, no es comúnmente capturada por trampas de captura viva (De Bondi et al. 2010).

Las trampas cámara son una herramienta de investigación ampliamente utilizada (Nichols et al. 2011; Díaz-Pulido y Payán Garrido 2012; Rovero et al. 2013; Meek et al.2014), debido a que perturban mínimamente el hábitat y al ser un método no invasivo, son ideales para el trabajo con especies amenazadas (Long et al. 2011; Trolliet et al. 2014). Este método también aporta información sobre la historia natural de las especies y las interacciones entre especies y el medio en el que habitan (Nyiramana et al. 2011; Sweitzer y Furnas 2016). El uso de las trampas cámara ha logrado importantes aportes al conocimiento científico sobre roedores, como ser la presencia, patrones de actividad y comportamiento de estos animales, la cual puede ser utilizadas como base científica en programas enfocados en su conservación (Yamada et al. 2010; Suzuki and Ando 2018; Rendall et al. 2014; McDonald et al. 2015; Figueroa-De-León et al. 2016; Hegerl et al. 2017).

Los datos obtenidos a través de foto-trampeo se pueden utilizar de múltiples maneras y pueden ayudar a entender varios aspectos de la ecología de las especies en estudio (O'Conell et al. 2011). Los modelos de ocupación son un ejemplo, a través de los cuales se puede inferir sobre la probabilidad de la presencia de la especie, abundancia relativa, determinar su uso de hábitat y poder definir su distribución potencial (MacKenzie et al. 2006; Carbone et al. 2002; Nichols et al. 2011; Díaz-Pulido y Payán Garrido 2012).

En Bolivia, el Programa para la Conservación de Grandes Carnívoros Andinos (PCCA), realizó campañas de fototrampeo en bosques secos del centro y sur de Bolivia, pertenecientes a la ecoregión "Boliviana-Tucumana" (Navarro y Maldonado 2005). A pesar de ser carnívoros medianos y grandes el principal objeto de estudio del PCCA, las trampas cámara lograron registrar la actividad de un amplio espectro de especies, entre ellas a A. boliviensis. Por ello, los objetivos de este trabajo fueron: demostrar la utilidad de registros fotográficos para generar datos sobre especies raras y difícilmente observables, usando a la rata chinchilla boliviana como ejemplo, y explorar la potencialidad de la aplicación de modelos de ocupación para la generación de información sobre este roedor endémico y críticamente amenazado.

\section{Materiales y Métodos}

Área de estudio. El estudio se realizó en dos áreas de bosques secos, que siguiendo la clasificación propuesta por Navarro y Maldonado (2005) e lbisch y Mérida (2003), pertenecen a la región "Boliviana-Tucumana" que se ubica en el centro y sur de Bolivia (Figura 1). El primer sitio de estudio está localizado en seis comunidades pertenecientes al municipio de San Lorenzo ( $\left.-21^{\circ} 11^{\prime} \mathrm{S},-64^{\circ} 27^{\prime} \mathrm{O}\right)$, ubicado al norte del departamento de Tarija. El segundo sitio de estudio corresponde al Área Natural de Manejo Integrado Municipal (ANMIM) “Lagarpampa -Mollepampa” (-18 $21^{\circ}$ 'S, $\left.-64^{\circ} 59^{\prime} \mathrm{O}\right)$, perteneciente al municipio de Aiquile al sur del departamento de Cochabamba.

Ambas áreas son zonas montañosas con rangos altitudinales de 1,000 hasta 2,500 msnm. La vegetación natural de estos bosques fue altamente modificada debido a la expansión de cultivos y la ganadería extensiva de ramoneo en libertad (Josse et al. 2009).

Análisis. Se utilizaron registros fotográficos obtenidos mediante campañas de foto-trampeo realizadas por el PCCA, por tanto, el diseño del estudio estuvo basado en la obtención de datos del oso andino (Tremarctos ornatus, Carnivora). Se colocaron 46 estaciones de foto-trampeo en el área de San Lorenzo, cubriendo un área de 289.15 km² y 15 en el ANMIM "Lagarpampa-Mollepampa", cubriendo un área de $112.75 \mathrm{~km}^{2}$. La distancia entre estaciones fue 1.5 km como mínimo. Es muy común que los estudios que utilizan el foto-trampeo se enfoquen en una sola especie, ignorando una potencial base de datos rica en información científica (Edwards 2018; Mazzamuto 2019). Si bien el oso andino presenta requerimientos ecológicos muy diferentes a los de $A$. boliviensis consideramos que los datos obtenidos de esta especie en el muestreo cumplen con los supuestos para la utilización de modelos de ocupación. Los supuestos determinados por MacKenzie et al. (2006) incluyen: 1) que la población en estudio sea cerrada, es decir, que no presente migraciones, muertes o nacimientos. Por dicha razón, se utilizaron datos obtenidos durante tres meses en época seca (agosto a octubre) y tres meses durante la época húmeda (enero a marzo). 2) que la toma de datos sea independiente, esto se logra por la distancia mínima entre cámaras, ya que siendo el área de acción de las especies altamente dependiente de su masa corporal (Tucker et al. 2014), creemos virtualmente imposible estar considerando al mismo individuo en dos estaciones de foto-trampeo. 3) que toda heterogeneidad se puede modelar, este supuesto se cumple ya que ambas áreas de estudio pertenecen a la misma ecorregión, presentan características similares y se muestrearon durante la misma época. Por tanto, las diferencias son detectables y fueron consideradas como covariables. 4) que no existan falsas detecciones, lo cual es fácilmente logrado porque las características morfológicas de $A$. boliviensis son particulares de la especie, lo cual hace que se la identifique correctamente. Asimismo, los modelos de ocupación, al calcular una probabilidad de detección, reducen posibles 


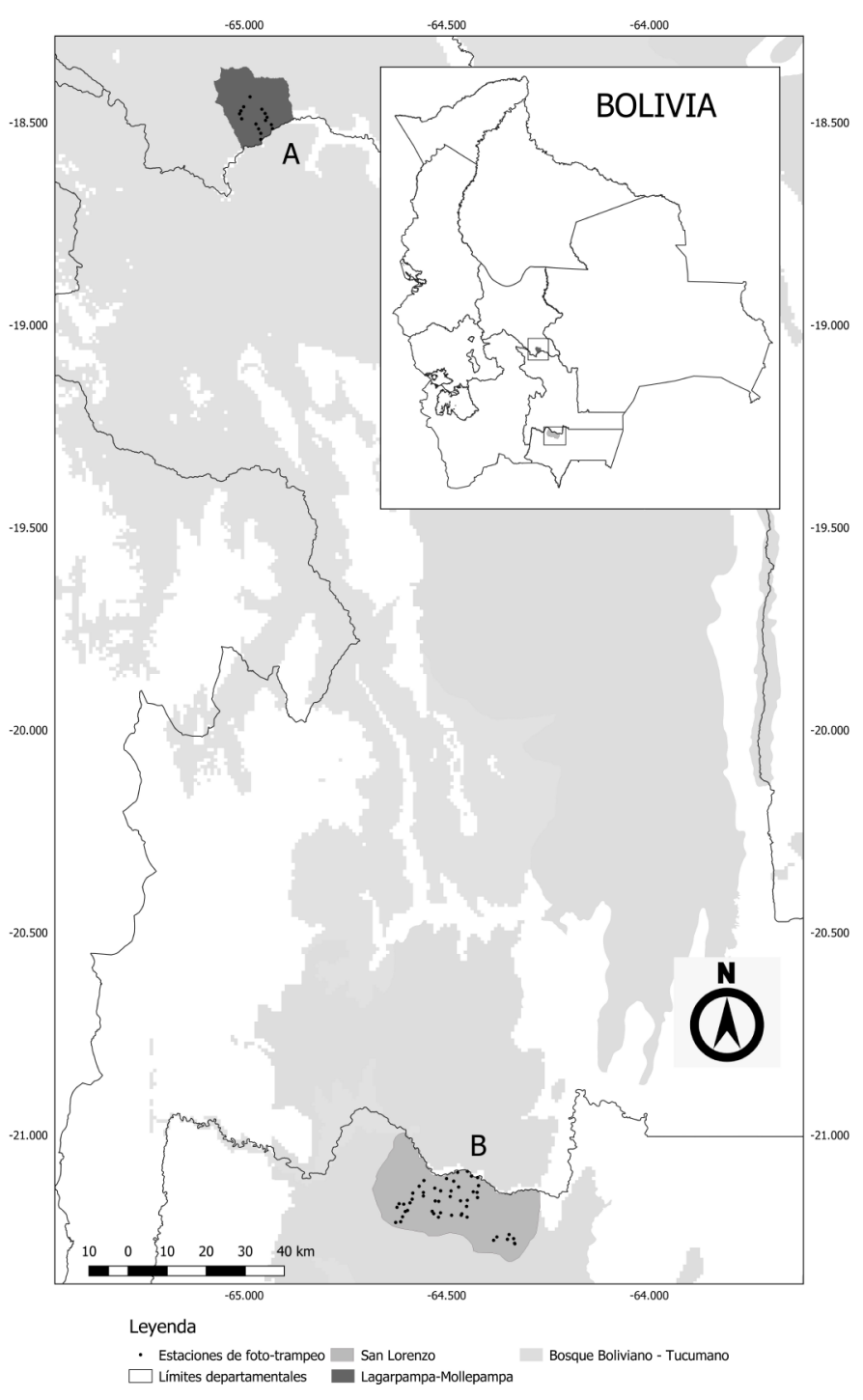

Figura 1. Mapa del área de estudio y localidad de las estaciones de foto-trampeo en Lagarpampa-Mollepampa, departamento de Cochabamba (A) y San Lorenzo, departamento de Tarija (B).

errores inducidos por la consideración de falsas ausencias, convirtiéndolos en una opción más atractiva frente a otras opciones de análisis (MacKenzie et al. 2006).

Una vez identificada la presencia de $A$. boliviensis, se verificaba la locación geográfica de la estación de foto-trampeo a la cual pertenecía la imagen, y el dato se incluía en una matriz de presencia-ausencia de la especie, llamada matriz de detección. Para realizar el análisis, se consideró una sola matriz de detección por época, la cual contenía las 61 estaciones de foto-trampeo como unidades de muestreo. Se procedió a un análisis exploratorio mediante el uso de modelos de ocupación a través del programa PRESENCE ( $\mathrm{v}$. 2.12.31; MacKenzie et al. 2006). Se escogieron cinco potenciales variables que se consideró podrían ser incorporadas en los modelos en este estudio, descritos a continuación:

Modelo de la trampa-cámara: se utilizaron dos tipos de cámaras diferentes, Cuddeback Ambush y Bushnell TrophyCam. Las que fueron colocadas de manera aleatoria en los diferentes sitios de muestreo, para ambas áreas consideradas. Debido a la sensibilidad, característica de cada modelo de cámara, se cree que ésta puede afectar la probabilidad de detectar especies de menor tamaño. Por tanto, se decidió utilizar el modelo como una variable de muestreo ya que potencialmente afecta la probabilidad de detección.

Hábitat alrededor de la cámara: considerando que los roedores tienen ámbitos de hogar reducidos, se consideró que el tipo de hábitat alrededor de la cámara es una variable que afectaría la probabilidad de presencia de la especie en el punto de muestreo. Se clasificó el hábitat de acuerdo a las características que ocupaban el $70 \%$ de la fotografía, excluyendo el nivel del suelo. Por ejemplo, si la fotografía presentaba roquedales en la mayoría de los alrededores se clasificaba como hábitat rocoso.

Sustrato: se consideró importante separar el sustrato de la variable "hábitat", puesto que éste parece estar más afectado por el cambio de época y uso por otros animales. Se clasificó al sustrato de acuerdo a las características presentadas por al menos el $70 \%$ del área cubierta por la fotografía a nivel del suelo. Por ejemplo, si el suelo en una fotografía presentaba mayormente helechos y otras plantas, se lo consideraba como un sustrato dominado por vegetación.

Elevación: en metros sobre el nivel del mar en el punto de muestreo.

Presencia de Leopardus geoffroyi: de acuerdo a lo observado en las fotografías analizadas, se observó que la presencia de esta especie y $A$. boliviensis parece estar fuertemente relacionada. Por tanto, si se encontraba al menos un registro fotográfico de este felino durante el periodo de muestreo para un sitio de muestreo, se lo consideraba como presente.

\section{Resultados}

Se obtuvieron 33 registros fotográficos en 14 estaciones para la época seca y 95 en 18 estaciones durante la época húmeda (Figura 2). La variación en elevación de dichos datos es 1,002 a 2,358 msnm. No existen registros previos de la presencia del género Abrocoma en la zona de estudio, por lo que nuestros datos son inéditos para el género. Las características morfológicas de los individuos fotografiados, cola larga y gruesa por ser peluda y las patas delanteras notorias además del tamaño en relación a otras especies y el tipo de hábitat, con roquedales abundantes y vegetación nativa, sugiere -sin lugar a dudas- de que los registros reportados en este trabajo corresponden a animales del género Abrocoma (Figura 2). Sobre la base de los bien conocidos patrones de distribución de otras especies del género y a las características morfológicas de los individuos fotografiados consideramos altamente confiable afirmar que estos animales corresponden a la especie Abrocoma boliviensis.

Con los datos obtenidos se procedió a construir modelos de ocupación, tres modelos para la época seca y tres modelos para la época húmeda (Tabla 1). Se dejó de considerar más modelos cuando éstos ya no convergieron y fueron ignorados en el análisis. Los resultados preliminares 


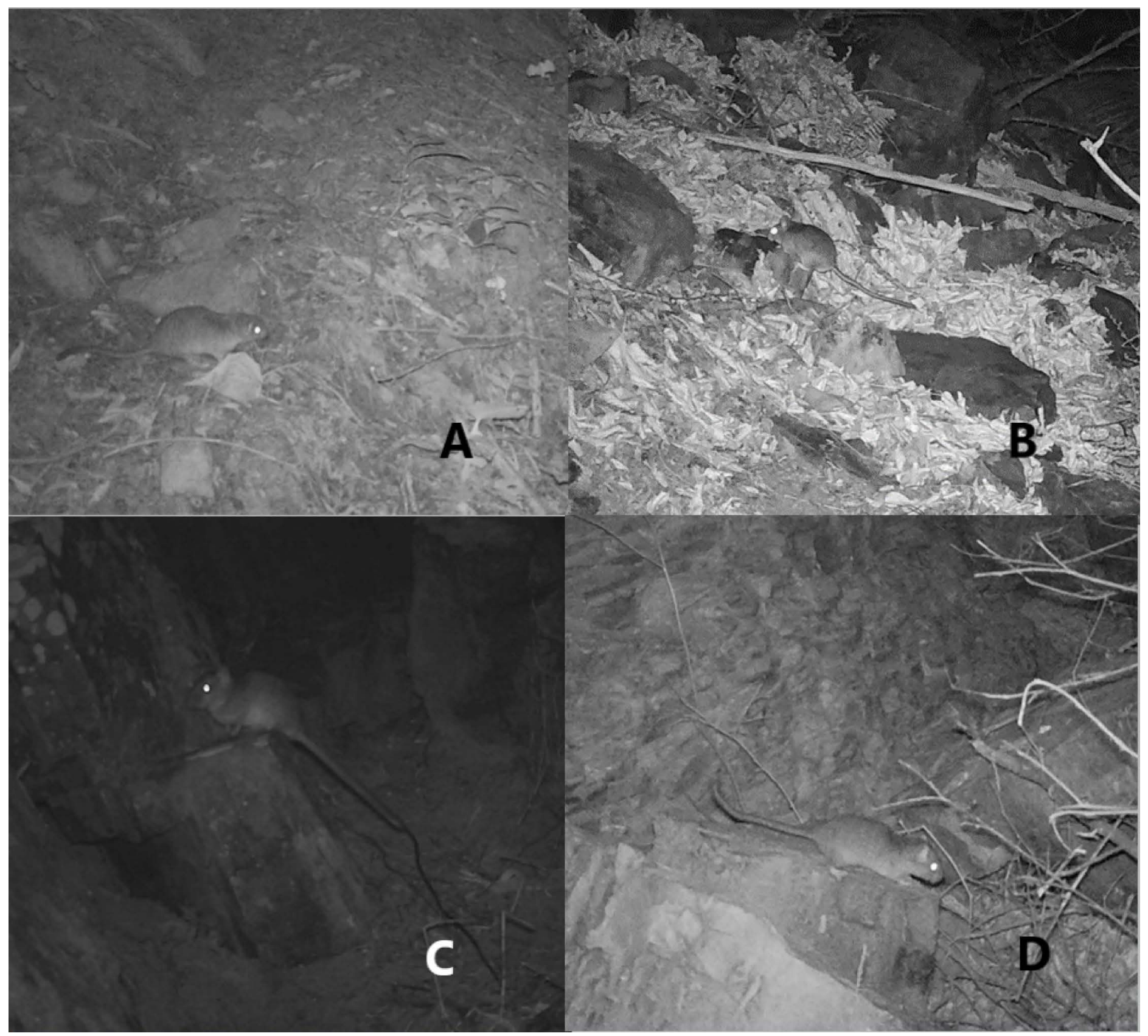

Figura 2. Registros de Abrocoma boliviensis en las trampas cámara. Donde (A) y (B) A. boliviensis se encuentra en un sustrato dominado por hojarasca, en la localidad de San Lorenzo y Lagarpampa-Mollepampa, respectivamente. Las figuras (C) y (D) muestran un sustrato dominantemente rocoso, en la localidad de San Lorenzo y Lagarpampa-Mollepampa, respectivamente.

determinan una probabilidad de ocupación ingenua (naïve occupancy) de 0.3023 para la época seca y 0.3396 para la época húmeda, las cuales nos sirve como base comparativa para demostrar las mejoras en los resultados obtenidos con una modelación integrando variables. Estos valores pueden ser interpretados como probabilidades de presencia de $30.23 \%$ y $33.96 \%$ de la especie en el área muestreada, comparable al $33 \%$ obtenido por Mazzamuto et al. (2019) en una investigación de similares características, con enfoque en el puercoespín crestado (Hystrix cristata, Hystricidae, Rodentia). Para poder explorar a mayor profundidad los factores que pueden influenciar la probabilidad que la especie esté presente y sea detectada, se procedió a correr los modelos que incluían las variables previamente mencionadas.
Al incorporar dichas variables en los modelos explorados, se pudo observar un ajuste en la probabilidad de ocupación de $A$. boliviensis, mostrando un potencial aumento de un $4 \%$ con respecto a la ocupación ingenua para ambas épocas (Tabla 1). Dentro de las variables exploradas para la época seca, se pudo observar que el modelo de la cámara utilizada es la variable de muestreo que influenció la probabilidad de detección de la especie (24 \% época seca y 36 \% época húmeda), siendo Bushnell TrophyCam la cámara con mejor rendimiento. En cuanto a las variables del sitio, el hábitat rocoso explica mejor la probabilidad de presencia de la especie. Por tanto, el mejor modelo correspondiente a la época seca, es aquel que ajusta la probabilidad de presencia de la especie de acuerdo al hábitat rocoso y al efecto del modelo de la cámara para la detección de la misma. 


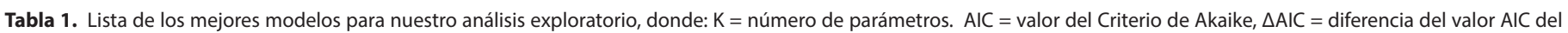
modelo con respecto al modelo con el menor valor para AIC, $w_{1}=$ peso del modelo, $\Psi=$ probabilidad de ocupación, $\rho=$ probabilidad de detección, $\mathrm{SE}=$ error estándar.

\begin{tabular}{|c|c|c|c|c|c|c|c|}
\hline & Modelo & $\mathbf{K}$ & AIC & $\Delta \mathrm{AIC}$ & $\mathbf{w}_{\mathbf{i}}$ & $\Psi$ & SE \\
\hline Época Seca & $\Psi$ (hábitat rocoso) $\rho()$. & 3 & 220.70 & 0.00 & 0.5500 & 0.3376 & 0.1022 \\
\hline \# detecciones = 33 & $\Psi(.) \rho$ (modelo de la cámara) & 3 & 221.54 & 0.84 & 0.3614 & 0.3848 & 0.0874 \\
\hline Naïve occupancy = 0.3023 & $\Psi(.) \rho()$. & 2 & 224.35 & 3.65 & 0.0887 & 0.3300 & 0.0799 \\
\hline Época Húmeda & $\Psi($ sustrato rocoso) $\rho()$. & 3 & 403.16 & 0.00 & 0.8367 & 0.3404 & 0.0787 \\
\hline$\#$ detecciones $=95$ & $\Psi$ (presencia predador) $\rho()$. & 3 & 406.47 & 3.31 & 0.1599 & 0.3400 & 0.0814 \\
\hline Naïve occupancy $=0.3396$ & $\Psi(.) \rho()$. & 2 & 414.13 & 10.97 & 0.0035 & 0.3402 & 0.0652 \\
\hline
\end{tabular}

Para la época húmeda, el sustrato rocoso y la presencia de su depredador fueron las variables con mayor efecto. En cuanto al hábitat y el sustrato, de acuerdo a lo esperado, la presencia de $A$. boliviensis parece estar positivamente relacionada a ambientes que presenten ya sea un sustrato y/o ambiente rocoso. Finalmente, la correlación entre la presencia de la especie en estudio y su predador principal podría dar pautas sobre la probabilidad de encontrar a este elusivo roedor en futuros diseños enfocados en el estudio de A. boliviensis.

\section{Discusión}

Para la región de Tarija, localizada en el sur de Bolivia, los presentes registros de una especie de rata chinchilla representarían los primeros y los más australes del país. En el área de Aiquile las fotografías de A. boliviensis representarían los primeros reportes para esta región en el departamento de Cochabamba. Si bien la utilización del foto-trampeo no es la técnica de preferencia para el estudio de animales de pequeño tamaño, este trabajo demostró su utilidad para la obtención de datos sobre roedores de difícil captura, como A. boliviensis. Los resultados obtenidos por De Bondi et al. (2010) también comprobaron la potencialidad de este método de muestreo, especialmente en la tasa de capturas y su utilidad en registrar especies elusivas. Además, es un método que permite realizar evaluaciones rápidas, determinando los atributos a los que una especie o un conjunto de ellas puedan deber su presencia (MacKenzie et al. 2006; Kalies et al. 2012).

Los registros de $A$. boliviensis obtenidos en este estudio en la región central de los Andes bolivianos refuerzan el conocimiento sobre la relación de la especie hacia ambientes con características rocosas, coincidiendo con las observaciones de Tarifa et al. (2009).

Respecto a los otros factores que difieren entre épocas debemos tratar por separado los aspectos relacionados al conocimiento de la especie y los factores metodológicos del muestreo. Primeramente, al ser la especie elusiva, principalmente con actividad nocturna y de porte pequeño presenta una baja detectabilidad que, sumada a la baja cantidad de registros obtenidos para cada época, no permiten construir modelos más complejos y por ende indagar en las variables que determinan la presencia de la especie en los bosques secos de la ecorregión Boliviana-
Tucumana. Portanto, cuandosetrataron deconstruirmodelos con más de una variable, estos no pudieron converger. Thomas et al. (2020) menciona la baja detectabilidad como limitante para el análisis y para la conducción a estimaciones sesgadas de las preferencias del hábitat (Ferreguetti et al. 2018b; Gorosito et al. 2018) sumado a una diferencia marcada entre épocas en el lugar de estudio (Chutipong et al. 2017). Por consiguiente, se considera necesario colectar datos futuros, aplicando un diseño específico para la especie, para explorar si se mejora la probabilidad de detección y genere mayor cantidad de datos.

Otro factor relacionado a la probabilidad de la presencia de $A$. boliviensis, son los registros del gato mediano Leopardus geoffroyi en la época húmeda. Este dato muestra la estrecha relación presa-predador, considerando que un roedor de tamaño considerable como A. boliviensis llegaría a ser la presa ideal en ambientes como los bosques BolivianoTucumanos o hábitats de la ladera oriental de los Andes centrales donde ambas especies confluyen (Anderson 1997; Noss et al. 2010). Probablemente esta variable mostró tener efecto solo en la época húmeda debido a la distancia a cuerpos de agua es una variable determinante para la presencia de felinos (Wilting et al. 2010). Ya que la marcada estacionalidad de estos bosques (Josse et al. 2009) presiona a este felino a quedarse en áreas más cercanas aguas permanentes durante la época seca y, por tanto, reducir el número de encuentros con A. boliviensis. Trabajos de trampas cámara dirigidos a esta interacción felino-roedor confirmarían aspectos ecológicas de estas especies (Suselbeek et al. 2014; Chutipong et al. 2017). Además, este resultado tiene un valor importante en futuros estudios de A. boliviensis, puesto que los indicios de L. geoffroyi podrían usarse como elementos para considerar sitios de muestreo $y$, posiblemente, aumentar la tasa de captura.

Analizando los aspectos metodológicos del muestreo se observa que, para la época seca, el factor tipo de cámara, está relacionado con la probabilidad de detectar la presencia de $A$. boliviensis, esto se debe a la sensibilidad que cada modelo de cámara presenta. De acuerdo a los resultados obtenidos se puede concluir que Bushnell TrophyCam tiene mayor éxito al registrar animales de menor envergadura. Muchos estudios resaltan que se deben considerar las características de diferentes modelos y marcas de cámaras, previo al diseño del muestreo (Rovero et al. 2013; 
Meek et al. 2014; McCleery et al. 2014; Thomas et al. 2020) Hay que recalcar que, si bien los datos usados han servido para mostrar la probabilidad de ocurrencia de este roedor, el diseño y disposición de las trampas cámara fueron diseñados para mamíferos grandes, objetivo de estudio del PCCA. Además, es importante considerar que las especies de menor tamaño presentan una menor tasa de captura (Tobler et al. 2008); sin embargo, y a pesar de no haber cumplido un diseño específico para la especie en estudio, se lograron obtener más de 100 registros de $A$. boliviensis, un número virtualmente imposible de obtener utilizando métodos de captura tradicionales.

Con el presente trabajo se demuestra que el uso de trampas cámara es una opción viable en el trabajo con mamíferos de porte mediano a pequeño. Los roedores, por su gran diversidad taxonómica y abundancia, tienen grupos únicos que atraviesan amenazas con altas tasas de extinción. Éstos aún tienen la necesidad de mayor conocimiento acerca de su rol en el ecosistema, valor como especie o importancia en comunidades locales. Las limitaciones en el muestreo con trampas de captura viva pueden ser complementadas con el uso de trampas cámara, optimizando esfuerzos de muestreo y maximizando el conocimiento científico (De Bondi et al. 2010; Edwards 2018). Los resultados obtenidos con este método pueden ayudar a definir el estado poblacional de pequeños mamíferos, también puede significar un método efectivo para monitorear la eficacia de las medidas de conservación aplicadas a estas especies. Por ello, para aumentar el conocimiento de la historia natural de Abrocoma boliviensis, especie elusiva, rara y en peligro crítico de extinción, se considera que futuros estudios deberían complementar métodos tradicionales de muestreo con foto-trampeo, para determinar probabilidad de presencia, uso de hábitat y patrones de distribución, y así desarrollar acciones de conservación con base científica.

\section{Agradecimientos}

Agradecemos al PCCA, PROMETA y el Museo de Historia Natural Alcide d'Orbigny por permitirnos utilizar los datos de foto-trampeo. A Whitley Fund for Nature, Chester Zoo, Darwin Innitiative, WildCRU, National Geographic Society y Phoenix Zoo, por dar los fondos que permitieron realizar los trabajos de campos. Por último, creemos que una de las mejores maneras de honrar el legado del Dr. Sydney Anderson, o "el profe" como muchos lo llamaban, es seguir contribuyendo al conocimiento científico de especies que él descubrió y estudió. Por tanto, este artículo es un agradecimiento a la dedicación y amor que el Dr. Anderson tenia por la mastozoología.

\section{Literatura citada}

Aguirre, L. F., R. Aguayo, J. A. Balderrama, C. Cortez, T. Tarifa, y O. Rocha (EDs.). 2009. Libro rojo de la fauna silvestre de vertebrados de Bolivia. Ministerio de Medio Ambiente y Agua. La Paz, Bolivia.
Anderson, S. 1997. Mammals of Bolivia: Taxonomy and Distribution. Bulletin of the American Museum of Natural History 231:652.

Bernal, N. 2016. Abrocoma boliviensis. The IUCN Red List of Threatened Species 2016:e.T18A22182349. http:// dx.doi.org/10.2305/IUCN.UK.2016-2.RLTS.T18A22182349 Consultado el 24 de mayo 2017.

Burgin, C. J., J. P. Colella, P. L. KahN, y N. S. Upham. 2018. How many species of mammals are there? Journal of Mammalogy 99:1-14.

Braun, J. K., y M. A. Mares. 1996. Unusual morphological and behavioral traits in Abrocoma (Rodentia: Abrocomidae) from Argentina. Journal of Mammalogy 77:891-897.

Braun, J.,K., y M. A. Mares. 2002. Systematics of the Abrocoma cinerea species complex (Rodentia: Abrocomidae), with a description of a new species of. Abrocoma. Journal of Mammalogy 83:1-19.

Carbone, C., S. Christie, K. Conforti, T. Coulson, N. Franklin, J. R. Ginsberg, M. Griffiths, J. Holden, M. Kinnaird, R. Laidlaw, A. Lynam, D. W. MacDonald, D. Martyr, C. McDougal, L. Nath, T. O'Brien, J. Seidensticker, J. L. D. Smith, R. Tilson, y W. N. Wan Shahrudin. 2002. The use of photographic rates to estimate densities of tigers and other cryptic mammals: response to Jennelle et al. Animal Conservation 5:121-123.

Chutipong, W., R. Steinmetz, T. Savini, y G. A. Gale. 2017. Assessing resource and predator effects on habitat use of tropical small carnivores. Mammal Research, 62:21-36.

De Bondi, N., J. G. White, M. Stevens, y R. Cooke. 2010. A comparison of the effectiveness of camera trapping and live trapping for sampling terrestrial small-mammal communities. Wildlife Research, 37:456-465.

Díaz-Pulido, A., y E. Payán Garrido. 2012. Manual de fototrampeo: una herramienta de investigación para la conservación de la biodiversidad en Colombia. Instituto de Investigaciones de Recursos Biológicos Alexander von Humboldt y Panthera. Bogotá, Colombia.

Edwards, S., S. Cooper, K. Uiseb, M. Hayward, B. Wachter, y J. Melzheimer. 2018. Making the most of by-catch data: Assesing the feasibility of utilizing non-target camera trap data for occupancy modelling of a large felid. African Journal od Ecology 56:885-894.

Emmons, L. H. 1999. A New Genus and Species of Abrocomid Rodent from Peru (Rodentia: Abrocomidae). Novitates American Museum of Natural History 3279:1-14.

Evolutionarily Distinct and Globally Endangered (EDGE). 2019. Abrocoma boliviensis. En: The EDGE Existence programme. https://www.edgeofexistence.org/what-is-edge/. Consultado el 4 de mayo 2019.

Ferreguetti, A. C., W. M. Tomas, y H. G. Bergallo. 2018 a. Patch occupancy and activity pattern of the spotted paca (Cuniculus paca Linnaeus, 1766) in a protected area of the Atlantic Forest, Brazil. Mammalia 83: 363-371.

Ferreguetti, A. C., W. M. Tomas, y H. G. Bergallo. 2018b. Density, habitat use, and daily activity patterns of the Red-rumped Agouti (Dasyprocta leporina) in the Atlantic Forest, Brazil. Studies on Neotropical Fauna and Environment 53:143-151.

Figueroa-De-León, A., E. J. Naranjo, H. Perales, A. SantosMoreno, y C. Lorenzo. 2016. Cavity occupation par plaine paca (Cuniculus paca) dans le Lacandon Rainforest, Chiapas, Mexique. Tropical Conservation Science 9:246-263. 
Glanz, W., y S. Anderson. 1990. Notes on Bolivian Mammals. A New Species of Abrocoma (Rodentia) and Relationships of the Abrocomidae. Novitates American Museum of Natural History 2991:1-32.

Gorosito, I. L., M. Marziali Bermúdez, y M. Busch. 2018. Advantages of combining generalized linear models and occupancy models to find indicators of habitat selection: Small mammals in agroecosystems as a case study. Ecological Indicators 85:1-10.

Grenyer, R., C. D. L. Orme, S. F. Jackson, G. H. Thomas, R. G. Davies, T. J. Davies, K. E. Jones, V. A. Olson, R. S. Ridgely, P. C. Rasmussen, T. S. Ding, P. M. Bennett, T. M. Blackburn, K. J. Gaston, J. L. Gittleman, y I. P. F. Owens. 2006. Global distribution and conservation of rare and threatened vertebrates. Nature 444:93-96.

GuZMÁn, J. A., y W. Sielfeld. 2011. A new northern distribution limit of Abrocoma bennettii (Rodentia, Abrocomidae) in the coastal Atacama desert, Paposo, north of Chile. Mastozoología Neotropical 18:131-134.

Hegerl, C., N. D. Burgess, M. R. Nielsen, E. Martin, M. Ciolli, y F. Rovero. 2017. Using camera trap data to assess the impact of bushmeat hunting on forest mammals in Tanzania. Oryx 51:87-97.

Hidalgo-Cossio, M., J. Salazar-Bravo, y T. Tarifa. 2016. Nuevas localidades en el centro de Bolivia para la especie endémica Abrocomaboliviensis (Rodentia:Abrocomidae) Mastozoología Neotropical 23:165-170.

InTERnATIONAL Institute fOR SPECIES ExPLORATION (IISE). 2011. State of Observed Species. Tempe AZ. http:/species.asu.edu/SOS. Consultado el 13 de noviembre del 2014.

IBISCH, P. L., y G. MéridA (eds.). 2003. Biodiversidad: La riqueza de Bolivia. Estado de conocimiento y conservación. Ministerio de Desarrollo Sostenible. Editorial FAN. Santa Cruz de la Sierra, Bolivia.

IsaAc, N. J. B., S. T. Turvey, B. Collen, C. Waterman y J. E. M. BAILliE. 2007. Mammals on the EDGE: Conservation priorities based on threat and phylogeny. Plos One 2:e296.

Josse C., F. Cuesta, G. Navarro, V. Barrena, E. Cabrera, E. Chacon-Moreno, W. erreira, M. Peralvo, J. Saito, y A. Tovar. 2009. Ecosistemas de los Andes del Norte y Centro. Bolivia, Colombia, Ecuador, Peru y Venezuela. Secretaría General de la Comunidad Andina. Lima, Perú.

Kalies, E. L., B. G. Dickson, C. L. Chambers, y W. W. Covington. 2012. Community occupancy responses of small mammals to restoration treatments in ponderosa pine forests, northern Arizona, USA. Ecological Applications. 22:204-217.

Law, B., T. Brassil, y L. Gonsalves. 2016. Recent decline of an endangered, endemic rodent: does exclusion of disturbance play a role for Hastings River mouse (Pseudomys oralis)? Wildlife Research 43:482.

Long, R.A., T. M. Donovan, P. MacKay, W. J. Zielinski, y J. S. Buzaset. 2011. Predicting carnivore occurrence with noninvasive surveys and occupancy modeling. Landscape Ecology, 26:327-340.

MacKenzie, D. I., J. D. Nichols, J. A. Royle, K. H. Pollock, L. L. Bailey, y J. E. Hines. 2006. Occupancy estimation and modeling: Inferring patterns and dynamics of species occurrence. Academic Press. London, England.
Mazzamuto, M.V., M. Lo Valvo, y S. Anile. 2019. The value od by-catch data: how species specific surveys can serve nontarget species. European Journal of Wildlife Research 65:68.

McCleery, R. A., C. L. Zweig, M. A. Desa, R. Hunt, W. M. Kitchens, y H. F. Percival. 2014. A novel method for cameratrapping small mammals. Wildlife Society Bulletin 38:887-891.

McDonald, P. J., A. D. Griffiths, C. E. M. Nano, C. R. Dickman, S. J. WARD, Y G. W. Luck. 2015. Landscape-scale factors determine occupancy of the critically endangered central rock-rat in arid Australia: The utility of camera trapping. Biological Conservation 191:93-100.

Meek, P. D., G. Ballard, A. Claridge, R. Kays, K. Moseby, T. O’Brien, A. O'Connell, J. Sanderson, D. E. Swann, M. Tobler, y S. Townsend. 2014. Recommended guiding principles for reporting on camera trapping research. Biodiversity and Conservation 23:2321-2343.

Murphy A. J., S. M. Goodman, Z. J. FarRIS, S. M. Karpanty, V. Andrianjakarivelo, y M. J. Kelly. 2017. Landscape trends in small mammal occupancy in the Makira-Masoala protected areas, northeastern Madagascar. Journal of Mammalogy 98:272-282.

Navarro, G., y M. Maldonado. 2005. Geografía Ecológica de Bolivia:Vegetación y Ambientes Acuáticos. Centro de Ecología y Difusión Simón I. Patiño. Santa Cruz de la Sierra Bolivia.

Nichols, J. D., K. U. Karanth, y A. F. O'Connell. 2011. Science, Conservation, and Camera Traps. Pp. 45-56 in Camera Traps in Animal Ecology: Methods and Analyses (O'Connell A. F., F. Allan, J. Nichols, D. Karanth, K. Ullas, eds.). Springer. New York, EE.UU. Noss, A., M. L. Villalba, y R. Arispe. 2010. Felidae. Pp. 401-444 in Distribución, Ecología y Conservación de los Mamíferos Medianos y Grandes de Bolivia (Wallace, R. B., H. Gómez, Z. R. Porcel, y D. I. Rumiz, eds.). Centro de Ecología y Difusión Simón I. Patiño. Santa Cruz de la Sierra, Bolivia.

Nyiramana, A., I. Mendoza, B. A. Kaplin, y P. M. Forget. 2011. Evidence for Seed Dispersal by Rodents in Tropical Montane Forest in Africa. Biotropica, 43:654-657.

O'Connell, A. F., J. D. Nichols, y K. U. Karanth. 2011. Camera Traps in Animal Ecology: Methods and Analyses. Springer. New York, EE.UU.

Pacheco, V. 2002. Mamíferos del Perú. Pp. 503-544 in Diversidad y Conservación de los Mamíferos Neotropicales (Ceballos, G., y J. A. Simonetti, eds.). CONABIO-UNAM. Ciudad de México, México.

Patton, J. L., U. F. J. Pardiñas, y G. D’Elía. 2015. Mammals of South America, Volume 2. The University of Chicago Press. Chicago, EE.UU.

Quinteros-Muñoz O. 2015. A new prey item for the snake Boiruna maculata (Serpentes: Dipsadidae) in the yungas of Bolivia. Phyllomedusa 14:79-81

Rendall, A. R., D. R. Sutherland, R. Cooke, y J. White. 2014. Camera Trapping: A Contemporary Approach to Monitoring Invasive Rodents in High Conservation Priority Ecosystems. Plos One 9:86592.

Rivero, K., D. Rumiz, J. Catari, H. Azurduy, O. Maillard, L. Acosta, M. Aponte, K. Osinaga, G. Soto, H. Saavedra, J. Aramayo, y L. Céspedes. 2004. Diagnóstico biológico preliminar y prioridades de investigación en el Área Protegida Municipal Parabanó. Revista Boliviana de Ecología y Conservación Ambiental 15:63-92. 
Rovero, F., F. Zimmermann, D. Berzi, y P. Meek. 2013. Which camera trap type and how many do I need? A review of camera features and study designs for a range of wildlife research applications. Hystrix 24:148-156.

Royle, J. A., y J. D. Nichols. 2003. Estimating abundance from repeated presence absence data or point counts. Ecology 84:777-790

Sweitzer, R. A., y B. J. Furnas. 2016. Data from camera surveys identifying co-occurrence and occupancy linkages between fishers (Pekania pennanti), rodent prey, mesocarnivores, and larger predators in mixed-conifer forests. Data in Brief 6:783-792.

Suselbeek, L., W. J. Emsens, B. T. Hirsch, R. Kays, J. M. Rowcliffe, V. Zamora-Gutierrez, y P. A. Jansen. 2014. Food acquisition and predator avoidance in a neotropical rodent. Animal Behaviour, 88:41-48.

SuzUKI, K. K., y M. ANDo. 2018. Early and efficient detection of an endangered flying squirrel by arboreal camera trapping. Mammalia 83:372-378.

Taraborelli, P., P. Sassi, M. Dacar, P. Moreno, y R. OJeda. 2015. Abrocoma schistacea (Rodentia: Abrocomidae). Mammalian Species 47:45-50.

Tarifa, T, C. Azurduy, R. Vargas, N. Huanca, J. Terán, G. Arriaran, C. Salazar, y L. Terceros. 2009. Observations on the natural history of Abrocoma sp. (Rodentia, Abrocomidae) in a Polylepis woodland in Bolivia. Mastozoología Neotropical 16:252-258.

Tarifa, T., y L. F. Aguirre. 2009. Mamíferos. Pp. 421-422 in Libro rojo de la fauna silvestre de vertebrados de Bolivia (Aguirre, L. F., R. Aguayo, J. A. Balderrama, C. Cortez, T. Tarifa y O. Rocha, eds.). Ministerio de Medio Ambiente y Agua. La Paz, Bolivia.

Thomas, M. L., L. Baker, J. R. Beattie, y A. M. BaKer. 2020. Determining the efficacy of camera traps, live capture traps, and detection dogs for locating cryptic small mammal species. Ecology and Evolution 10:1054-1068.

Tobler, M. W., S. E. Carrillo-Percastegui, R. Leite Pitman, R. Mares, y G. Powell. 2008. An evaluation of camera traps for inventorying large- and medium-sized terrestrial rainforest mammals. Animal Conservation 11:169-178.

Trolliet, F., M. C. Huynen, C. Vermeulen, y A. Hambuckers. 2014. Use of camera traps for wildlife studies. A review. Biotechnology Agronomy Society and Enviromental 18:446-454.

TuCKer, M. A., T. J. ORd, y T. L. Rogers. 2014. Evolutionary predictors of mammalian home range size: body mass, diet and the environment. Global Ecology and Biogeography 23:1105-1114.

ÚbedA, C. A., D. E. Grigera, y A. R. RecA. 1994. Conservación de la Fauna de Tetrápodos. II. Estado de Conservación de los Mamíferos del Parque y Reserva Nacional Nahuel Huapi. Mastozoología Neotropical 1:29-44.

Wilting, A., A. Cord, A. J. Hearn, D. Hesse, A. Mohamed, C. Traeholdt, S. Cheyne, S. Sunarto, M. A. Jayasilan, J. Ross, A.C. Shapiro, A. Sebastian, S. Dech, C. Breitenmoser, J. Sanderson, J. W. Duckworth, y H. Hofer. 2010. Modelling the species distribution of Flat-headed cats (Prionailurus planiceps), an endangered South-East Asian small felid. Plos One 5:e9612.

Yamada, F., N. Kawauchi, K. Nakata, S. Abe, N. Kotaka, A. Takashima, C. Murata, y A. Kurolwa. 2010. Rediscovery After Thirty Years Since the Last Capture of the Critically Endangered Okinawa Spiny Rat Tokudaia muenninki in the Northern Part of Okinawa Island. Mammal Study 35:243-255.
Associated editor: Mariana Díaz

Submitted: July 6, 2020; Reviewed: July 25, 2020;

Accepted:September 3, 2020; Published on line: September 13, 2020. 